\title{
Alopecia Areata and Habit Tic Deformities
}

\author{
Marina Isabel Horne ${ }^{a} \quad$ Joyce B. Utzig ${ }^{b}$ Evan A. Rieder ${ }^{c}$ Colombina Vincenzi $^{d}$ \\ Antonella Tosti ${ }^{\mathrm{e}}$ \\ a Private Office, Salvador, Brazil; ${ }^{\mathrm{b}}$ Federal University of Southern Border, Passo Fundo, Brazil; ${ }^{\mathrm{c}}$ The Ronald O. Perelman \\ Department of Dermatology, New York School of Medicine, New York, NY, USA; ${ }^{\mathrm{d}}$ Department of Specialised \\ Experimental and Diagnostic Medicine, Dermatology, Alma Mater Studiorum - Università di Bologna, Bologna, Italy; \\ e Departament of Dermatology and Cutaneous Surgery, University of Miami Miller School of Medicine, Miami, FL, USA
}

\section{Established Facts}

- Alopecia areata is associated with nail disorders in a large number of patients. It is well documented by the occurrence of nail pittings, trachyonychia, and onychorrehxis.

\section{Novel Insights}

- We describe the association of alopecia areata and habit tic nail disorder in two patients. As far as we are concerned, it is a new finding related to psychological stress in alopecia areata patients.

\section{Keywords}

Alopecia areata · Nail · Nail disorder · Psychological stress · Quality of life

\section{Abstract}

Nail involvement is a very common finding in patients with alopecia areata (AA). Most commonly evidenced by nail pitting, nail abnormalities can affect up to $66 \%$ of AA patients. We present two cases of the unusual association between habit tic nail deformities and AA.

(c) 2018 S. Karger AG, Basel

\section{Introduction}

Nail abnormalities occur in 10-66\% of patients with alopecia areata (AA), depending on the study cited. Kasumagic-Halilovic and Prohic [1] examined the prevalence of nail changes in 200 patients with AA. They found nail changes in $24.5 \%$ of patients. The most common finding was nail pitting, accounting for $79.6 \%$ of all AA nail abnormalities. Other nail changes included trachyonychia (14.3\%) and onychorrhexis (6.1\%). Nail changes were more frequent in patients with severe forms of AA $[1,2]$.

\section{KARGER}

(c) 2018 S. Karger AG, Basel

E-Mail karger@karger.com

www.karger.com/sad
Marina Isabel Horne

Av. Tancredo Neves, 1632, Ed. Salvador Trade Center Torre Sul

sala 1812 - Caminho das Árvores

Salvador, Bahia 40295-010 (Brazil)

E-Mail marinahorne@hotmail.com 


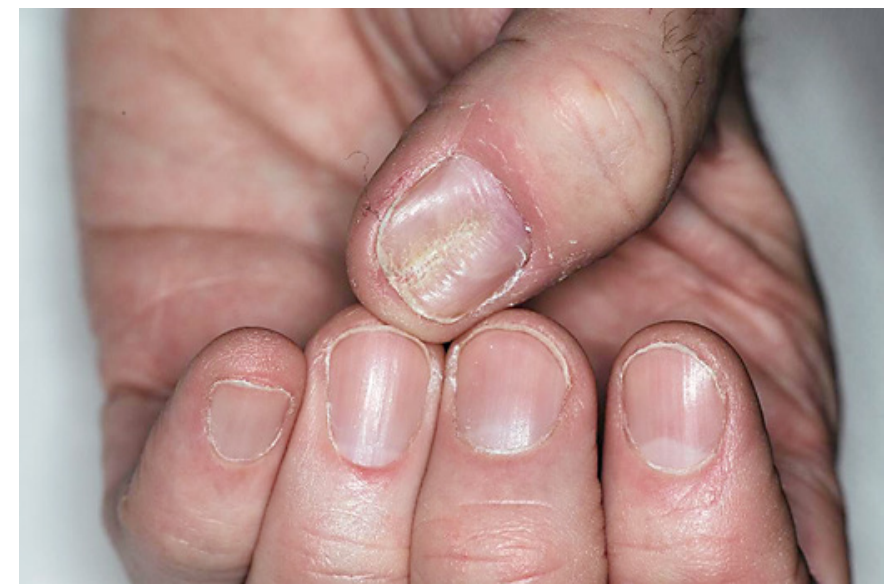

Fig. 1. Longitudinal groove and horizontal ridging of both thumb nails caused by repeated trauma.

Habit tic deformity is a self-induced form of nail dystrophy caused by external trauma to the nail matrix [3]. This process is thought to occur from the repetitive manipulation of the nail plate and matrix of the thumb by the adjacent second digit. Most commonly the habit tic deformity symmetrically affects the thumbnails, although any nail can be involved $[3,4]$. The deformity tends to be more prominent on the dominant hand [4]. On examination, the nail plate presents with a central longitudinal groove, extending from the proximal nail fold to the distal margin, with multiple horizontal ridges. Enlargement of the lunula and absence of cuticle can be seen in more severe cases $[3,4]$.

We report two patients with AA and nail abnormalities due to habit tic damage to the nail apparatus.

\section{Case Report}

Case Report 1

A 55-year-old man presented with a 5-month history of severe scalp hair loss. Physical examination revealed alopecia affecting more than $60 \%$ of his scalp, consistent with an ophiasis pattern of AA. Digital exam demonstrated typical habit tic deformity of both thumb nails, characterized by the aforementioned longitudinal groove and horizontal ridging (Fig. 1). He admitted that he was repeatedly traumatizing his nails and related the habit to the stress caused by his hair loss.

\section{Case Report 2}

A 30-year-old man presented for evaluation of 10 years of diffuse loss of hair. Physical examination demonstrated total loss of scalp and body hair, consistent with alopecia universalis. Nail examination revealed a longitudinal depression involving the lateral nail plate of the first digit of both hands. The depression involved

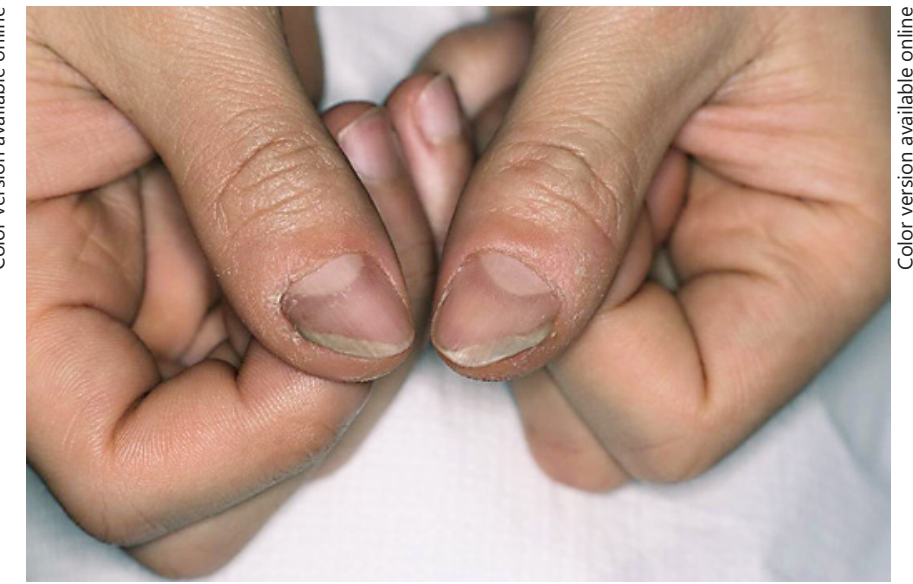

Fig. 2. Longitudinal depression involving the lateral nail plate and enlargement of the lunula and onycholysis of the lateral portion of the nail plate.

$2 / 3$ of the nail plate without reaching the free margin of the nail plate. Enlargement of the lunula and onycholysis of the lateral portion of the nail plate were also evident (Fig. 2). When questioned, the patient admitted to pushing and compressing the lateral part of the nail and at the same time lifting the nail plate of his thumbs using his second finger. He related this behavior to the anxiety caused by his alopecia.

\section{Discussion}

We present two cases of patients with alopecia areata presenting habit tic nail deformities. One patient had the typical median nail plate deformity, while the other patient demonstrated a lateral deformity induced by trauma. This lateral phenotype is caused by repeated compression of the lateral side of the nail instead of the proximal nail fold. Both patients admitted to repetitive manipulation of the nail plate and related their behaviors to the stress caused by their disfiguring hair disorders.

Given the clinical and behavioral history elicited from the two case patients, the most likely etiology for the habit tic is psychosocial stress. We may hypothesize that severe, widespread, and appearance-altering hair loss may have led to emotional distress. Instead of being verbalized through feelings, patient distress may have been manifested through an anxious habit, such as repetitive nail unit traumatization. An association between AA and psychological distress, including depressive and anxious tendencies, is well known [5]. In addition, AA may impair quality of life by altering self-perception and self-esteem, thus interfering with social interactions. 
Although the clinical phenotype of isolated habit tic deformity and AA-associated habit tic deformity is similar, it is important to distinguish isolated self-induced nail changes from nail changes associated with $\mathrm{AA}$, as the prognoses and possible treatments may be different. Treatments for AA may include corticosteroids or immunomodulators. It is possible that with resolution or improvement of AA and its psychological stress, the accompanying habit tic deformity may subside. Habit tic deformity generally improves when the external trauma is abolished. This can be reached using physical barriers, such as adhesive tape or krazy glue applied directly to the nail fold, or with behavioral therapy [6]. N-acetyl cysteine or antidepressant selective serotonin reuptake inhibitors may be effective as habit tic deformity may be related to obsessive-compulsive behaviors. However, evidence for these modalities in the treatment of self-induced nail disorders is lacking [7].
In summary, we describe two cases of severe AA associated with habit tic deformity. To our knowledge, these are the first two cases to describe this clinical association, which we believe to have evolved as the result of alopeciarelated psychological stress. Dermatologists should always consider the possibility of AA in patients that present with median nail dystrophy.

\section{Statement of Ethics}

The authors declare compliance to guidelines for human research and that all subjects have given their informed consent to publishing their details and photographs.

\section{Disclosure Statement}

The authors declare no conflict of interest. There was no funding support for this research.

\section{References}

\footnotetext{
1 Kasumagic-Halilovic E, Prohic A: Nail changes in alopecia areata: frequency and clinical presentation. J Eur Acad Dermatol Venereol 2009;23:240-241.

-2 Tosti A, Morelli R, Bardazzi F, Peluso AM: Prevalence of nail abnormalities in children with alopecia areata. Pediatr Dermatol 1994; 11:112-115.

3 Perrin AJ, Lam JM: Habit-tic deformity. CMAJ 2014;186:371.
}

4 Norton LA: Self-induced trauma to the nails. Cutis 1987;40:223-227.

5 Tosti A: Practice gaps. Alopecia areata and comorbid conditions. JAMA Dermatol 2013; 149:794.

6 Ring DS: Inexpensive solution for habit-tic deformity. Arch Dermatol 2010;146:1222-1223.

7 Rieder EA, Tosti A: Onychotillomania: an underrecognized disorder. J Am Acad Dermatol 2016;75:1245-1250. 